\title{
Self-monitoring of blood glucose compared to self-monitoring of urine glucose in adults with newly diagnosed Type 2 diabetes receiving structured education: a cluster randomised controlled trial
}

Running title: Self-monitoring of blood glucose versus urine glucose

HM Dallosso (University Hospitals of Leicester, Leicester Diabetes Centre)

$\mathrm{DH}$ Bodicoat (University of Leicester, Diabetes Research Centre)

M Campbell (University of Sheffield, School of Health and Related Research)

ME Carey (University Hospitals of Leicester, Leicester Diabetes Centre)

MJ Davies (University of Leicester, Diabetes Research Centre)

HC Eborall (University of Leicester, Department of Health Sciences)

M Hadjiconstantinou (University of Leicester, Diabetes Research Centre)

K Khunti (University of Leicester, Diabetes Research Centre)

J Speight (AHP Research, UK; The Australian Centre for Behavioural

Research in Diabetes; Deakin University, Centre for Mental Health and

Wellbeing Research, Australia)

S Heller (University of Sheffield, Department of Human Metabolism)

Corresponding Author: Helen Dallosso, helen.dallosso@uhl-tr.nhs.uk

Word count: $3077 \quad$ Abstract: 244;

Trial registration: ISRCTN 95696668

Funding: The study was funded by a grant from Diabetes UK (BDA: RD 05/3233). The funder approved the study design but was not involved in the running of the study, collection and analysis of data or preparation of manuscript.

Conflicts of interest: MJD has acted as consultant, advisory board member and speaker for Novartis, Novo Nordisk, Sanofi-Aventis, Lilly, Merck Sharp \& Dohme, Boehringer Ingelheim and Roche and received grants in support of investigator and investigator initiated trials from Novartis, Novo Nordisk, Sanofi-Aventis, Lilly, Pfizer, Merck Sharp \& Dohme and GlaxoSmithKline. KK has acted as consultant, advisory board member for Novo Nordisk, Eli Lilly, Merck Sharp \& Dohme, Bristol Myers Squibb, Roche and received payment for lectures from Novo Nordisk, Eli Lilly, Sanofi-Aventis, Novartis, Merck Sharp \& Dohme, Janssen, Astra Zeneca, Boehringer Ingelheim Ltd. JS has received consultancy fees from Roche Diagnostics Australia, and grants and consultancy fees from Sanofi Diabetes. SH reports personal fees from Lifescan. Other authors have nothing to declare.

\section{Novelty statement}

- This is the first pragmatic trial comparing blood glucose monitoring with urine glucose monitoring within an established and widely available structured education course.

- Equivalent improvements in $\mathrm{HbA1c}$ and other biomedical outcomes were seen regardless of the mode of monitoring, though participants showed a slight preference towards blood monitoring. 
- Promoting urine monitoring as the method of choice in structured education programmes for the newly diagnosed might lead to substantial savings without impairing outcomes. However those who find blood monitoring more useful should be permitted to do so, but would need to provided with support by their healthcare team. 


\section{Abstract}

Aims: To compare the effectiveness and acceptability of self-monitoring of blood glucose and urine glucose in adults with newly diagnosed Type 2 diabetes.

Methods: Multi-site cluster randomised controlled trial with practice-level randomisation. Participants attended a group structured education programme which included a module on self-monitoring using blood glucose or urine glucose monitoring. $\mathrm{HbA} 1 \mathrm{c}$ and other biomedical measures as well as psychosocial data were collected at 6, 12 and 18 months. A total of 292 people with Type 2 diabetes were recruited.

Results: HbA1c reduced significantly from baseline to 18 months in both the blood monitoring $(-12 \mathrm{mmol} / \mathrm{mol}[\mathrm{SE}=2 \mathrm{mmol} / \mathrm{mol}] ;-1.1 \%$ [SE $=0.2 \%])$ and urine monitoring $(-13 \mathrm{mmol} / \mathrm{mol}[\mathrm{SE}=2 \mathrm{mmol} / \mathrm{mol}] ;-1.2 \%$ [SE $=0.2 \%])$ groups, with no difference between groups (difference adjusted for cluster effect and baseline value $=-1 \mathrm{mmol} / \mathrm{mol}[95 \% \mathrm{Cl}=-3,2] ;-0.1 \%[95 \% \mathrm{Cl}=-0.3,0.2])$. Similar improvements were observed for the other biomedical outcomes, with no differences between groups. Both groups showed improvements in total treatment satisfaction, generic well-being, and diabetes-specific well-being, and a less threatening view of diabetes, with no differences between groups at 18 months. Approximately 1 in 5 participants in the urine monitoring arm switched to blood monitoring, while those in the blood monitoring arm rarely switched $(18 \%$ vs $1 \%$ at 18 months; $p<0.001)$.

Conclusions: People with newly diagnosed Type 2 diabetes who attended structured education showed comparable improvements in HbA1c at 18 
months, regardless of whether they were assigned to blood or urine selfmonitoring. 


\section{Introduction}

Self-monitoring of blood glucose is a core component of effective selfmanagement of Type 1 diabetes, and of Type 2 diabetes for people using insulin or sulphonylureas[1,2]. However, the benefit is less clear for people with Type 2 diabetes using diet or oral agents that do not increase the risk of hypoglycaemia[3-7]. This lack of clarity is in part because the trials conducted are heterogeneous, differing in trial design, population recruited, and how the use of self-monitoring was implemented[3-7]. As a result, many propose that the evidence does not justify self-monitoring of blood glucose by people with non-insulin treated Type 2 diabetes, and that the costs saved could be redirected to evidence-based management strategies[8]. Others acknowledge that implementing self-monitoring in isolation without training of both patients and clinicians may waste resources but maintain that including it within a structured approach to management may be effective and clinically relevant $[9,10]$.

Very few studies have examined self-monitoring of blood glucose in people with newly diagnosed Type 2 diabetes[11,12] or compared it with other forms of glucose monitoring (e.g. urine monitoring) which might be provided at less cost[13-15]. We hypothesised that for adults with newly diagnosed type 2 diabetes attending group structured education, use of self-monitoring of urine glucose would be as effective as blood glucose monitoring, in terms of improving and sustaining glycaemic control over 18 months. We tested this hypothesis within the context of the DESMOND (Diabetes Education SelfManagement for Ongoing and Newly diagnosed Diabetes) structured 
education programme[16]. The standard curriculum was modified with modules on the two methods of self-monitoring added, enabling comparison in a randomised controlled trial, while ensuring that both arms experienced comparable structured education in self-management.

\section{Methods}

The study design and protocol have been published elsewhere[17]. In brief, the study was an 18-month multi-site cluster-randomised controlled trial with practices randomised to one of two arms. All participants attended a DESMOND structured education programme[16], which included a module on either blood or urine self-monitoring. Follow-up data were collected at 6,12 and 18 months post-education. Ethical approval was received from a Research Ethics Committee (07/H0304/129) and local research governance approval from participating health trusts.

\section{Setting and participants}

Sixty-five general practices (from inner city to rural settings) in England referred adults with Type 2 diabetes to the study within 12 weeks of diagnosis. They were excluded if they were under 18 years, using insulin, had severe and enduring mental health problems, were not primarily responsible for their own care, were unable or unwilling to participate in a group programme or were taking part in another research study. Participants were required to attend the DESMOND programme within six months of diagnosis and were not eligible if they had already started regular self-monitoring of blood glucose. 


\section{Randomisation}

The study was cluster-randomised, with randomisation at practice level, after stratification for site and practice list size. The study was unblinded but practices agreed to participate prior to randomisation and were not informed of their study arm allocation. However, it is likely that they became aware over time as participants attended the practice for ongoing clinical support and repeat prescriptions for monitoring resources. Practice staff were instructed to refer patients without telling them which arm they had been allocated to and patients were not informed of their study arm allocation until they had given informed consent.

\section{Study intervention}

Participants attended the DESMOND 'Newly Diagnosed' programme[16]. This is delivered by two trained and accredited educators as a single 6-hour session or two 3-hour sessions one week apart, and usually includes a 20minute session covering both urine and blood self-monitoring. For the study, the time spent discussing self-monitoring was extended to 100 minutes, and the course was delivered in two sessions, one week apart. The development, content, and piloting of the new self-monitoring modules are described in the protocol paper[17]. The approach to self-monitoring was non-directive, but promoted the practice of self-monitoring as a tool to support decision-making in regard to lifestyle changes and medication use. Participants were encouraged to discover when and how often to monitor, as well as how to interpret results and explore options for change. Although participants were 
allocated to blood or urine self-monitoring, they were free to change their method of monitoring or to stop monitoring at any time. During the study, they obtained further monitoring supplies by requesting a prescription from their practice. Most participants were exempt from prescription charges and the small number liable to pay, were refunded any charges they incurred. Practices were also refunded the prescribing costs to prevent cost being a barrier to self-monitoring or to participation in the trial.

The primary outcome was mean glycated haemoglobin (HbA1c) at 18 months. The secondary outcomes included biomedical and psychosocial variables, measured at 6, 12 and 18 months. Biomedical measures included lipid profile, blood pressure, body weight and waist circumference. Psychosocial processes and outcomes were measured using questionnaires covering various aspects of diabetes and self-monitoring. Satisfaction with treatment was assessed using the Diabetes Treatment Satisfaction Questionnaire[18], which measures: 1) 'total treatment satisfaction' 2) 'perceived frequency of hyperglycaemia' and 3) 'perceived frequency of hypoglycaemia'. Psychological well-being was measured using the 28-item Well-Being Questionnaire[18], from which two 12-item constructs were produced: 1) 'generic well-being' and 2) 'diabetes-specific well-being'. Perceptions of diabetes were measured using a diabetes-specific version of the Brief IIIness Perceptions Questionnaire[19]. For all measures, a higher score indicates more of the construct being measured. 
The trial was designed as a non-inferiority trial with the aim of demonstrating a mean equivalence in $\mathrm{HbA} 1 \mathrm{c}$ at 18 months of within $5 \mathrm{mmol} / \mathrm{mol}( \pm 0.5 \%)$. Assuming, at 18 months, a conservative standard deviation of $16 \mathrm{mmol} / \mathrm{mol}$ (1.5\%)[20], 80\% power and 5\% significance, 142 participants were required per arm, increasing to 163 per arm to allow for clustering, assuming an intracluster correlation of 0.05 and mean cluster size of four. Assuming $20 \%$ nonconsent and $20 \%$ drop-out, numbers required were 254 referrals and 204 consenting per arm.

Baseline characteristics were summarised by group at each follow-up point as was the monitoring method that the participant reported using. For each outcome and follow-up, the mean (standard error) change from baseline at each follow-up was estimated by arm and compared using generalised estimating equations adjusted for baseline value and a term that accounted for the practice-level clustering. These equations assumed an exchangeable correlation matrix, robust standard errors and a normal distribution for the data, except for the reported monitoring method, which used a Binomial distribution. The primary analyses followed the intention-to-treat principle and data were analysed according to randomisation arm, regardless of whether participants continued to use that monitoring method or provided follow-up data. Missing data were replaced using multiple imputation, which accounted for the clustering as far as possible except where this meant that the model would not converge (waist circumference, systolic and diastolic blood pressure, HDL and LDL cholesterol and W-BQ28 'generic well-being', because the models that accounted for site did not converge). 
Analyses were repeated in two key subsets: 1)only those with complete data available, i.e. with no imputation of missing data, and 2)only those who were using their randomised monitoring method at the follow-up time point of interest. The latter is analogous to a per protocol analysis in an individually randomised trial and, thus, is referred to as 'per protocol' from herein for ease of understanding. All p-values are two-sided and statistical significance was assessed at the $5 \%$ level. Analyses were conducted in Stata 12.1. Data are presented as estimate [ $95 \%$ confidence interval].

\section{Results}

The participant flow is shown in Figure 1. A total of 75 general practices were recruited and randomised; 37 to blood monitoring and 38 to urine monitoring. The blood monitoring practices referred 253 people, of whom 140(55\%) consented to join the study. The urine monitoring practices referred 275 people, of whom 152(55\%) joined the study (Fig 1). The main reasons for non-participation were no longer interested in taking part, booked but did not attend, unable to arrange a suitable date and unable to contact. The baseline characteristics of those who consented are shown in Table 1. There was no significant difference within randomisation groups in age, gender or baseline $\mathrm{HbA} 1 \mathrm{c}$ in those who did and did not provide biomedical data at 18 months. Participants who did not complete the questionnaire at 18 months were younger than respondents in both arms (blood:p<0.001; urine:p=0.05) but did not differ in terms of HbA1c (blood: $p=0.45$; urine: $p=0.74$ ) or gender (blood: $p=0.12$; urine: $p=0.71$ ). 
Results for the biomedical outcomes are shown in Table 2. There was a significant reduction in $\mathrm{HbA} 1 \mathrm{c}$ from baseline in the blood monitoring group of $13(\mathrm{SE}=2) \mathrm{mmol} / \mathrm{mol}(1.2 \%(\mathrm{SE}=0.2 \%))$ at 6 months, of $12(2) \mathrm{mmol} / \mathrm{mol}$ $(1.1 \%(0.2 \%))$ at 12 months, and of $12.0(2.2) \mathrm{mmol} / \mathrm{mol}(1.1 \%(0.2 \%)$ at 18 months. Likewise, there was a significant reduction in the urine monitoring group of $15(2) \mathrm{mmol} / \mathrm{mol}(1.4 \%(0.2 \%))$ at 6 months, of $14(2) \mathrm{mmol} / \mathrm{mol}$ $(1.3 \%(0.2 \%))$ at 12 months, and of $13(2) \mathrm{mmol} / \mathrm{mol}(1.2 \%(0.2 \%))$ at 18 months. There were no significant differences between groups in terms of HbA1c change at each follow-up (Table 2).

Likewise, there were improvements from baseline in body mass index, waist circumference, weight, systolic and diastolic blood pressure, and total and LDL cholesterol in both monitoring groups at each follow-up (most of which were statistically significant, with no significant differences between groups at each follow-up (Table 2). Sensitivity and per protocol analyses showed no significant differences between the two groups for any of the biomedical outcomes (Supplementary Table 1).

In both groups, there were improvements from baseline in total treatment satisfaction, generic well-being, diabetes-specific well-being, and diabetes was perceived as less threatening; most of which were statistically significant (Table 3). There were no significant changes in perceived frequency of hyperor hypoglycaemia. The only significant difference between the groups was that at 6 months, people randomised to urine monitoring perceived diabetes 
as less threatening than those randomised to blood monitoring (difference = $2.8 ; 95 \% \mathrm{Cl}=-5.3,-0.3)$.

Sensitivity and per protocol analyses (Supplementary Table 2) show that many of the previously non-significant differences between groups strengthened and became statistically significant. When only complete data were analysed (i.e.missing data not imputed), the urine monitoring group were less satisfied with their treatment, had better generic and diabetes-specific well-being, and a less threatening view of diabetes, for at least one follow-up time point. In per protocol analyses, the urine monitoring group reported lower perceived frequency of hyperglycaemia and a less threatening view of diabetes at 6 months.

Participants randomised to blood monitoring were more likely to be using their randomised monitoring method than urine monitoring participants at 6 months ( $86 \%$ vs $74 \%, p=0.04), 12$ months ( $85 \%$ vs $69 \%, p=0.01)$, and 18 months (79\% vs 59\%, p<0.01) (Fig 2). However, the proportion of participants who did not monitor at all was not significantly different between the groups ( 6 months: $12 \%$ vs $8 \%, p=0.14 ; 12$ months: $12 \%$ vs $14 \%, p=0.70 ; 18$ months: $17 \%$ vs $20 \%, p=0.58)$. Rather, those initially randomised to urine monitoring tended to switch to blood monitoring whilst those initially randomised to blood monitoring almost never switched method ( 6 months $12 \%$ vs $1 \%, p=0.01 ; 12$ months: $11 \%$ vs $0 \%, p<0.001 ; 18$ months: $18 \%$ vs $1 \%, p<0.01)$. In both groups, participants who were monitoring were most likely to be doing so 1 to 3 times per week (data not shown). Half of the participants were prescribed metformin 
during the study. This usually happened at diagnosis ( $48 \%$ by 6 months) with small increases in prescription rates occurring over time $(50 \%$ and $54 \%$ at 12 and 18 months). There were no differences between the two arms.

\section{Discussion}

Both groups showed significant improvements in $\mathrm{HbA} 1 \mathrm{c}$ and other biomedical and psychosocial outcomes during the study, but there no significant differences in changes between the groups, indicating that blood monitoring provided no additional benefit when compared with urine monitoring.

Our study had a robust, multi-site cluster design, to prevent contamination between groups, and a clinically relevant follow-up period of 18 months. It was conducted in a primary care setting, with broad inclusion criteria; it involved attending a structured education intervention that is delivered widely in the UK, and participants were recruited using a standard referral pathway. The pragmatic study design therefore embeds it in the real world and provides good external validity. Validated generic and diabetes-specific questionnaires were used in the evaluation. The two groups were well matched, and the fact that both received the same amount of education enabled us to control for effects of education. Although eligibility for referral did not include a minimum $\mathrm{HbA} 1 \mathrm{c}$ level, the mean value at baseline of $65 \mathrm{mmol} / \mathrm{mol}(8.1 \%)$ was high enough to show improvement. This has been a weakness of other studies where a low baseline HbA1c may have caused a 'floor effect' [12,21,22]. 
The study limitations include a lower consent rate than anticipated (although rates were identical in each arm), which resulted in not recruiting to target. However, sample size calculations were based on a predicted standard deviation in $\mathrm{HbA} 1 \mathrm{c}$ at the end of the study of $(16 \mathrm{mmol} / \mathrm{mol}(1.5 \%))$, while the actual standard deviation $(11 \mathrm{mmol} / \mathrm{mol}(1.0 \%))$ was lower than this. Substituting the observed SD into the sample size calculation and keeping other assumptions the same, the required sample size would be 73 participants per arm, indicating that the study was adequately powered to meet its primary objective. The low consent rate was due to logistical problems that commonly occur in standard clinical pathways (e.g.difficulty contacting patients, cancellation and non-attendance). The level of data return dropped from $91 \%$ at 6 months to $71 \%$ at 18 months. However there were no significant differences at baseline in age, gender or $\mathrm{HbA} 1 \mathrm{c}$ between those who did and did not provide follow-up data, and sensitivity analysis suggests that level of data return did not have a large impact on results. The information and support that participants received for self-monitoring was almost exclusively from the DESMOND course. Although healthcare professionals in participating practices received a training visit before the trial[17], and were asked to provide impartial support and advice on selfmonitoring during the study, their input was unlikely to have been large. Furthermore some participants in the qualitative sub-study (Eborall, Submitted to Diabetic Medicine) reported a lack of support from healthcare professionals with interpreting results and in some cases disapproval of the method. Thus, the full potential of blood monitoring to effect changes in self-management may have been limited. 
Systematic reviews and meta-analyses[3-7], as well as recently published studies not included in the reviews[23-26], have generally reported little benefit in biomedical outcomes of self-monitoring of blood glucose in people with non-insulin treated Type 2 diabetes with one exception. When the approach to monitoring is "structured", with participants following clearly defined monitoring schedules and receiving regular feedback and advice from trained healthcare professionals, then clinically relevant improvements in glycaemic control have been observed[23-26]. However, studies of "structured" self-monitoring are efficacy trials and cannot be compared closely with our trial, which was pragmatic, conducted in primary care and involved a single session of group education with relatively little input from healthcare professionals at practice level. The DiGEM study[22] also conducted in primary care in the UK reported no difference in $\mathrm{HbA} 1 \mathrm{c}$ at 12 months in patients with established Type 2 diabetes randomised to a control group (no monitoring) or one of two blood monitoring groups in which one received education and feedback from trained practice nurses.

Only two recent trials have evaluated self-monitoring of blood glucose in people with newly diagnosed Type 2 diabetes[11,12] and these showed conflicting results. In the ESMON study[11], there were large but similar improvements in $\mathrm{HbA} 1 \mathrm{c}$ over 12 months in both groups $(21 \mathrm{mmol} / \mathrm{ml}(1.9 \%))$ in those who used blood monitoring and $(19 \mathrm{~mol} / \mathrm{mol}) 1.7 \%)$ ) in those who did not monitor), while in the St Carlos Study[12], the group using self-monitoring showed greater "remission" and "regression" compared with the group that did 
not monitor. In both studies, referral took place in hospital clinics and the interventions included substantial amounts of one-to-one education with healthcare professionals as well as quarterly $\mathrm{HbA} 1 \mathrm{c}$ tests, which were used to inform changes in medication. Fundamental differences in design therefore make it difficult to compare the results of these trials with our own.

In our study, the proportion switching methods or stopping monitoring altogether was greater in the urine monitoring group who tended to report less satisfaction with their treatment. The qualitative sub-study (Eborall, Submitted to Diabetic Medicine) has helped explain this: interviewees reported blood monitoring as accurate, convenient and useful in a practical sense for managing their diabetes, while many initially positive views of urine monitoring lessened over time as interviewees came to find it inaccurate. This s supported by previous qualitative work which reported a perception of urine monitoring as inconvenient and unhygienic[27].

With regard to psychosocial outcomes, those randomised to urine monitoring in our study had a tendency toward better generic and diabetes-specific wellbeing at follow-up, consistent with trials reporting that emotional well-being may be adversely affected by blood monitoring, including increased anxiety[11,28] and depressive symptoms[11,29]. In addition, those using urine monitoring in the present study reported a less threatening view of diabetes (at 6 months). Our qualitative sub-study suggested that urine monitoring provided insufficient visibility of diabetes to interviewees, leading some to question their diagnosis (Eborall, Submitted to Diabetic Medicine). In contrast 
blood monitoring provided a more visible reminder - both of having diabetes and of the effects of activities on blood glucose - which may have raised the level of threat perceived, echoing previous research where blood monitoring led to anxiety and self-blame in participants whose glucose readings were high[30].

We conclude that in the context of the present study, which reflects current practice in the UK, urine monitoring, as part of structured self-management education, is as effective as blood monitoring over the first 18 months after diagnosis. Many participants found urine monitoring useful and acceptable and if this method of self-monitoring was made available as the method of choice at diagnosis then it might lead to substantial cost savings without impairing outcomes. Nevertheless, the significant number who chose to switch to blood monitoring suggests that many found this more useful. These individuals should be permitted to do, but would need to be provided with ongoing advice and support by their healthcare team. 


\section{Funding}

The study was funded by a grant from Diabetes UK (BDA: RD 05/3233). The funder approved the study design but was not involved in the running of the study, collection and analysis of data or preparation of manuscript.

\section{Conflicts of interest}

MJD has acted as consultant, advisory board member and speaker for Novartis, Novo Nordisk, Sanofi-Aventis, Lilly, Merck Sharp \& Dohme, Boehringer Ingelheim and Roche and received grants in support of investigator and investigator initiated trials from Novartis, Novo Nordisk, Sanofi-Aventis, Lilly, Pfizer, Merck Sharp \& Dohme and GlaxoSmithKline. KK has acted as consultant, advisory board member for Novo Nordisk, Eli Lilly, Merck Sharp \& Dohme, Bristol Myers Squibb, Roche and received payment for lectures from Novo Nordisk, Eli Lilly, Sanofi-Aventis, Novartis, Merck Sharp \& Dohme, Janssen, Astra Zeneca, Boehringer Ingelheim Ltd. JS has received consultancy fees from Roche Diagnostics Australia, and grants and consultancy fees from Sanofi Diabetes. SH reports personal fees from Lifescan. Other authors have nothing to declare.

Acknowledgements The authors would like to acknowledge: Heather Daly and Lorraine Martin-Stacey who wrote the curriculum (with input from the authors) and trained the Educators; the Educators who were trained and then delivered the intervention; the practice nurses who referred participants and collected the follow-up data; and the people with Type 2 diabetes who participated in this study. $\mathrm{HD}, \mathrm{DB}, \mathrm{MC}, \mathrm{MD}, \mathrm{MH}$, and $\mathrm{KK}$ acknowledge support from the National Institute for Health Research Collaboration for Leadership in Applied Health Research and Care - East Midlands (NIHR CLAHRC - EM), the Leicester Clinical Trials Unit and the NIHR Leicester-Loughborough Diet, Lifestyle and Physical Activity Biomedical Research Unit which is a partnership between University Hospitals of Leicester NHS Trust, Loughborough University and the University of Leicester.

Author Contributions: SH, MJD, KK, HMD, MEC and JS were involved in study conception and design; HMD and $\mathrm{MH}$ managed the study; $\mathrm{DB}, \mathrm{MC}$ 
provided statistical input and analysis; HMD, SH, JS, HE and DB were involved in drafting the paper. All authors contributed to editing and approved the final text. 


\section{References}

1. The Diabetes Control and Complications Trial Research Group. The effect of intensive treatment of diabetes on the development and progression of long-term complications in insulin-dependent diabetes mellitus. $\mathrm{N}$ Engl J Med 1993 Sep 30;329(14):977-986.

2. Naik RG, Ellis SL. Self-monitoring of blood glucose in insulin-requiring type 2 diabetes. Diabetes Technology and Therapeutics 200801 Jun 2008;10(SUPPL. 1):S67-S71.

3. Clar C, Barnard K, Cummins E, et al. Self-monitoring of blood glucose in type 2 diabetes: systematic review. Health Technology Assessment 2010;14(12):1-140.

4. Malanda UL, Welschen LMC, Riphagen II, et al. <br />Self-monitoring of blood glucose in patients with type 2 diabetes mellitus who are not using insulin(Review). Health technology assessment 2012.

5. Farmer AJ, Perera R, Ward A, et al. Meta-analysis of individual patient data in randomised trials of self monitoring of blood glucose in people with noninsulin treated type 2 diabetes. BMJ 2012;344:e486.

6. Towfigh A, Romanova M, Weinreb JE, et al. Self-monitoring of blood glucose levels in patients with type 2 diabetes mellitus not taking insulin: a meta-analysis. American Journal of Managed Care 2008;14(7):468-475.

7. Davidson MB. Evaluation of self monitoring of blood glucose in non-insulintreated diabetic patients by randomized controlled trials: little bang for the buck. Reviews on Recent Clinical Trials 2010;5(3):138-142.

8. Malanda UL, Bot SD, Nijpels G. Self-Monitoring of Blood Glucose in Noninsulin-Using Type 2 Diabetic Patients: It is time to face the evidence. Diabetes Care 2013 January 01;36(1):176-178.

9. Polonsky WH, Fisher L. Self-Monitoring of Blood Glucose in NoninsulinUsing Type 2 Diabetic Patients: Right answer, but wrong question: selfmonitoring of blood glucose can be clinically valuable for noninsulin users. Diabetes Care 2013 January 01;36(1):179-182.

10. Speight J, Browne JL, Furler J. Challenging evidence and assumptions: is there a role for self-monitoring of blood glucose in people with type 2 diabetes not using insulin? Curr Med Res Opin 2013 03/01; 2013/10;29(3):161-168.

11. O'Kane MJ, Bunting B, Copeland M, et al. Efficacy of self monitoring of blood glucose in patients with newly diagnosed type 2 diabetes (ESMON study): randomised controlled trial. BMJ 2008;336(7654):1174-1177. 
12. Duran A, Martin P, Runkle I, et al. Benefits of self-monitoring blood glucose in the management of new-onset Type 2 diabetes mellitus: The St Carlos Study, a prospective randomized clinic-based interventional study with parallel groups. Journal of Diabetes 2010;2(3):203-211.

13. Allen BT, DeLong ER, Feussner JR. Impact of glucose self-monitoring on non-insulin-treated patients with type II diabetes mellitus. Randomized controlled trial comparing blood and urine testing. Diabetes Care 1990;13(10):1044-1050.

14. Fontbonne A, Billault B, Acosta $M$, et al. Is glucose self-monitoring beneficial in non-insulin-treated diabetic patients? Results of a randomized comparative trial. Diabetes \& metabolism 1989;15(5):255-260.

15. Gallichan M. Self-monitoring by patients receiving oral hypoglycaemic agents: A survey and a comparative trial. Practical Diabetes International 1994;11(1):28-30.

16. Davies MJ, Heller S, Skinner TC, et al. Effectiveness of the diabetes education and self management for ongoing and newly diagnosed (DESMOND) programme for people with newly diagnosed type 2 diabetes: Cluster randomised controlled trial. BMJ 200801 Mar 2008;336(7642):491495.

17. Dallosso H, Eborall H, Daly H, et al. Does self monitoring of blood glucose as opposed to urinalysis provide additional benefit in patients newly diagnosed with type 2 diabetes receiving structured education? The DESMOND SMBG randomised controlled trial protocol. BMC Family Practice 2012;13(1):18.

18. Bradley C. The Well-Being Questionnaire. In: Bradley C, editor. Handbook of Psychology and Diabetes Switzerland: Harwood Academic Publishers; 1994.

19. Weinman J, Petrie KJ, Moss-Morris R, et al. The Illness Perceptions Questionnaire: a new method for assessing the cognitive presentation of illness. Psychology \& Health 1996;11:431-441.

20. Khunti K, Gray LJ, Skinner T, et al. Effectiveness of a diabetes education and self management programme (DESMOND) for people with newly diagnosed type 2 diabetes mellitus: three year follow-up of a cluster randomised controlled trial in primary care. BMJ 2012;344:e2333.

21. Scherbaum WA, Ohmann $\mathrm{C}$, Abholz $\mathrm{H}$, et al. Effect of the frequency of self-monitoring blood glucose in patients with type 2 diabetes treated with oral antidiabetic drugs - a multi-centre, randomized controlled trial. PLoS ONE 2008;3(8):e3087. 
22. Farmer A, Wade A, Goyder E, et al. Impact of self monitoring of blood glucose in the management of patients with non-insulin treated diabetes: open parallel group randomised trial. BMJ 2007;335:132.

23. Polonsky WH, Fisher L, Schikman $\mathrm{CH}$, et al. Structured Self-Monitoring of Blood Glucose Significantly Reduces A1C Levels in Poorly Controlled, Noninsulin-Treated Type 2 Diabetes: Results from the Structured Testing Program study. Diabetes Care 2011 February 01;34(2):262-267.

24. Bosi E, Scavini M, Ceriello A, et al. Intensive Structured Self-Monitoring of Blood Glucose and Glycemic Control in Noninsulin-Treated Type 2 Diabetes: The PRISMA randomized trial. Diabetes Care 2013 October 01;36(10):28872894.

25. Kempf K, Tankova T, Martin S. ROSSO-in-praxi-international: Long-Term Effects of Self-Monitoring of Blood Glucose on Glucometabolic Control in Patients with Type 2 Diabetes Mellitus Not Treated with Insulin. Diabetes Technology \& Therapeutics 2013;15(1):89-96.

26. Franciosi M, Lucisano G, Pellegrini $F$, et al. ROSES: role of selfmonitoring of blood glucose and intensive education in patients with Type 2 diabetes not receiving insulin. A pilot randomized clinical trial. Diabetic Med 2011;28(7):789-796.

27. Lawton J, Peel E, Douglas M, et al. 'Urine testing is a waste of time': newly diagnosed Type 2 diabetes patients' perceptions of self-monitoring. Diabetic Medicine 2004;21(9):1045-1048.

28. Franciosi M, Pellegrini F, De Berardis G, et al. The Impact of Blood Glucose Self-Monitoring on Metabolic Control and Quality of Life in Type 2 Diabetic Patients: An urgent need for better educational strategies. Diabetes Care 2001 November 01;24(11):1870-1877.

29. Fisher L, Polonsky W, Parkin CG, et al. The impact of blood glucose monitoring on depression and distress in insulin-naïve patients with type 2 diabetes. Current Medical Research \& Opinion 2011;27(S3):39-46.

30. Peel E, Parry O, Douglas M, et al. Diagnosis of type 2 diabetes: a qualitative analysis of patients' emotional reactions and views about information provision. Patient Educ Couns 2004 6;53(3):269-275. 
Table 1. Baseline characteristics of study participants by monitoring group.

\begin{tabular}{|c|c|c|}
\hline Variable & $\begin{array}{c}\text { Blood } \\
\text { monitoring } \\
(N=140)\end{array}$ & $\begin{array}{c}\text { Urine } \\
\text { monitoring } \\
(\mathrm{N}=152)\end{array}$ \\
\hline \multicolumn{3}{|l|}{ Demographic factors } \\
\hline N (\%) Women & $69(49.3)$ & $65(42.8)$ \\
\hline N (\%) White European & 124 (93.9) & $121(87.1)$ \\
\hline Age, years & $57.1(11.3)$ & $59.4(11.6)$ \\
\hline \multicolumn{3}{|l|}{ Biomedical measures } \\
\hline $\mathrm{HbA} 1 \mathrm{c}, \mathrm{mmol} / \mathrm{mol}$ & $65(22)$ & $66(23)$ \\
\hline $\mathrm{HbA} 1 \mathrm{c}, \%$ & $8.1(4.2)$ & $8.2(4.3)$ \\
\hline Total cholesterol, mmol/l & $5.3(1.2)$ & $5.3(1.4)$ \\
\hline HDL cholesterol, mmol// * & $1.2(0.4)$ & $1.2(0.3)$ \\
\hline LDL cholesterol, $\mathrm{mmol} / /^{*}$ & $3.0(1.1)$ & $3.1(1.1)$ \\
\hline Systolic blood pressure, $\mathrm{mm} / \mathrm{Hg}$ & $135.5(15.1)$ & $136.2(15.6)$ \\
\hline Diastolic blood pressure, $\mathrm{mm} / \mathrm{Hg}$ & $81.5(10.1)$ & $81.4(12.1)$ \\
\hline Weight, kg & $94.4(20.2)$ & $91.8(18.5)$ \\
\hline Waist circumference, $\mathrm{cm}^{*}$ & $107.3(16.1)$ & $106.1(15.6)$ \\
\hline $\mathrm{BMI}, \mathrm{kg} / \mathrm{m}^{2}$ & $34.2(10.8)$ & $32.8(7.9)$ \\
\hline \multicolumn{3}{|l|}{ Psychosocial measures } \\
\hline \multicolumn{3}{|l|}{ DTSQs } \\
\hline Total treatment satisfaction * (score range: $0-36)$ & $25.8(8.0)$ & $24.5(8.4)$ \\
\hline Perceived frequency of hyperglycaemia* (score range: $0-6$ ) & $1.8(1.9)$ & $1.6(1.8)$ \\
\hline Perceived frequency of hypoglycaemia* (score range: $0-6$ ) & $0.9(1.4)$ & $0.9(1.3)$ \\
\hline \multicolumn{3}{|l|}{$W-B Q 28$} \\
\hline 12-item generic well-being (score range: $0-36$ ) & $21.7(6.7)$ & $23.2(6.6)$ \\
\hline Diabetes-specific well-being (score range: $0-36$ ) & $23.9(7.2)$ & $24.8(7.1)$ \\
\hline \multicolumn{3}{|l|}{$B I P Q$} \\
\hline Threatening view of diabetes * (score range: $0-80$ ) & $35.1(12.8)$ & $33.8(12.1)$ \\
\hline
\end{tabular}

Data are mean (standard deviation).

Abbreviations: DTSQ, Diabetes Treatment Satisfaction Questionnaire; W-BQ28, Well-Being Questionnaire; BIPQ, Brief Illness Perceptions Questionnaire.

*More than $20 \%$ of the data for these variables were missing. For all variables, missing values were imputed prior to data analysis (see Methods). 
Table 2. Mean changes from baseline for biomedical outcomes by monitoring group $(n=292)$

\begin{tabular}{|c|c|c|c|}
\hline \multirow[b]{2}{*}{ Variables } & \multicolumn{2}{|c|}{$\begin{array}{c}\text { Mean (SE) change } \\
\text { from baseline } \\
\end{array}$} & \multirow{2}{*}{$\begin{array}{c}\text { Difference }(95 \% \mathrm{Cl}) \\
\text { between } \\
\text { monitoring groups }\end{array}$} \\
\hline & Blood monitoring & Urine monitoring & \\
\hline \multicolumn{4}{|c|}{$\mathrm{HbA} 1 \mathrm{c}, \mathrm{mmol} / \mathrm{mol}$} \\
\hline 6 months & $13(2) * * *$ & $15(2) * * \star$ & $0(-3,2)$ \\
\hline 12 months & $12(2) * * *$ & $15(2)$ *** & $0(-3,2)$ \\
\hline 18 months $^{b}$ & $12(2) * * *$ & $13(2)$ *** & $-1(-3,2)$ \\
\hline \multicolumn{4}{|l|}{$\mathrm{HbA} 1 \mathrm{c}, \%$} \\
\hline 6 months & $-1.20(0.16)^{\star * *}$ & $-1.40(0.19)^{\star \star *}$ & $-0.01(-0.23,0.22)$ \\
\hline 12 months & $-1.08(0.18)^{\star * *}$ & $-1.33(0.20)^{\star * *}$ & $-0.02(-0.27,0.24)$ \\
\hline 18 months $^{b}$ & $-1.13(0.21)^{\star * *}$ & $-1.20(0.20)^{\star * *}$ & $-0.05(-0.28,0.18)$ \\
\hline \multicolumn{4}{|l|}{$\mathrm{BMI}, \mathrm{kg} / \mathrm{m}^{2}$} \\
\hline 6 months & $-1.28(0.21)^{\star * *}$ & $-1.03(0.29)^{\star \star *}$ & $0.17(-0.49,0.82)$ \\
\hline 12 months & $-1.04(0.34)^{\star \star}$ & $-0.86(0.23)^{\star \star *}$ & $0.38(-0.34,1.10)$ \\
\hline 18 months & $-0.80(0.43)$ & $-1.06(0.42)^{\star}$ & $-0.64(-1.59,0.31)$ \\
\hline \multicolumn{4}{|c|}{ Waist circumference, $\mathrm{cm}$} \\
\hline 6 months & $-1.99(1.15)$ & $-2.87(0.96)^{\star *}$ & $-0.54(-2.42,1.33)$ \\
\hline 12 months & $-2.97(2.88)$ & $-1.80(1.73)$ & $-0.55(-3.96,2.86)$ \\
\hline 18 months & $-3.07(3.42)$ & $-2.98(1.52)$ & $0.01(-2.58,2.61)$ \\
\hline \multicolumn{4}{|l|}{ Weight, kg } \\
\hline 6 months & $-3.59(0.70)^{* * *}$ & $-2.97(0.73)^{\star \star \star}$ & $0.02(-1.87,1.92)$ \\
\hline 12 months & $-2.86(0.95)^{\star \star}$ & $-2.80(0.65)^{\star \star *}$ & $-0.17(-2.29,1.94)$ \\
\hline 18 months & $-1.45(1.28)$ & $-3.62(1.25)^{\star \star}$ & $0.12(-3.09,3.33)$ \\
\hline \multicolumn{4}{|c|}{ Systolic BP, mmHg } \\
\hline 6 months & $-2.49(1.67)$ & $-4.11(1.68)^{*}$ & $-0.96(-4.41,2.49)$ \\
\hline 12 months & $-4.39(2.22)$ & $-4.20(2.06)^{*}$ & $1.05(-2.13,4.24)$ \\
\hline 18 months & $-1.60(2.30)$ & $-3.98(1.83)^{*}$ & $-0.41(-3.36,2.55)$ \\
\hline \multicolumn{4}{|c|}{ Diastolic BP, mmHg } \\
\hline 6 months & $-3.32(1.10)^{\star *}$ & $-3.54(0.93)^{* * *}$ & $-0.75(-2.55,1.05)$ \\
\hline 12 months & $-4.88(1.22)^{\star * *}$ & $-3.37(1.18)^{\star *}$ & $0.53(-1.52,2.59)$ \\
\hline 18 months & $-2.56(1.52)$ & $-3.63(2.63)$ & $-0.63(-3.07,1.81)$ \\
\hline \multicolumn{4}{|c|}{ Total cholesterol, $\mathrm{mmol} / \mathrm{l}$} \\
\hline 6 months & $-0.77(0.13)^{* * *}$ & $-0.98(0.13)^{\star \star *}$ & $-0.20(-0.46,0.06)$ \\
\hline 12 months & $-1.04(0.15)^{\star * *}$ & $-0.99(0.14)^{\star * *}$ & $-0.12(-0.35,0.12)$ \\
\hline 18 months & $-1.02(0.14)^{\star * *}$ & $-0.97(0.15)^{\star * *}$ & $-0.07(-0.27,0.12)$ \\
\hline \multicolumn{4}{|c|}{ HDL cholesterol, mmol/l } \\
\hline 6 months & $-0.02(0.05)$ & $0.03(0.03)$ & $0.02(-0.04,0.08)$ \\
\hline 12 months & $0.07(0.03)^{*}$ & $0.14(0.10)$ & $0.03(-0.07,0.14)$ \\
\hline 18 months & $0.02(0.03)$ & $0.01(0.03)$ & $0.00(-0.05,0.06)$ \\
\hline \multicolumn{4}{|c|}{ LDL cholesterol, mmol// } \\
\hline 6 months & $-0.57(0.12)^{* * *}$ & $-0.69(0.15)^{\star * *}$ & $0.03(-0.28,0.22)$ \\
\hline 12 months & $-0.90(0.18)^{* * *}$ & $-0.83(0.16)^{\star * *}$ & $0.01(-0.19,0.21)$ \\
\hline 18 months & $-0.67(0.19)^{\star \star}$ & $-0.57(0.17)^{\star *}$ & $-0.01(-0.17,0.15)$ \\
\hline \multicolumn{4}{|c|}{ 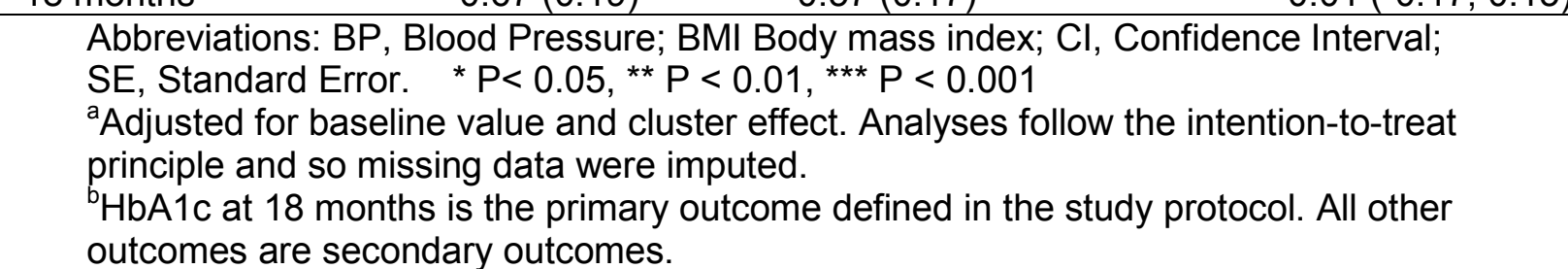 } \\
\hline
\end{tabular}


Table 3.Mean changes from baseline for psychosocial outcomes by monitoring group $(\mathrm{N}=292)$.

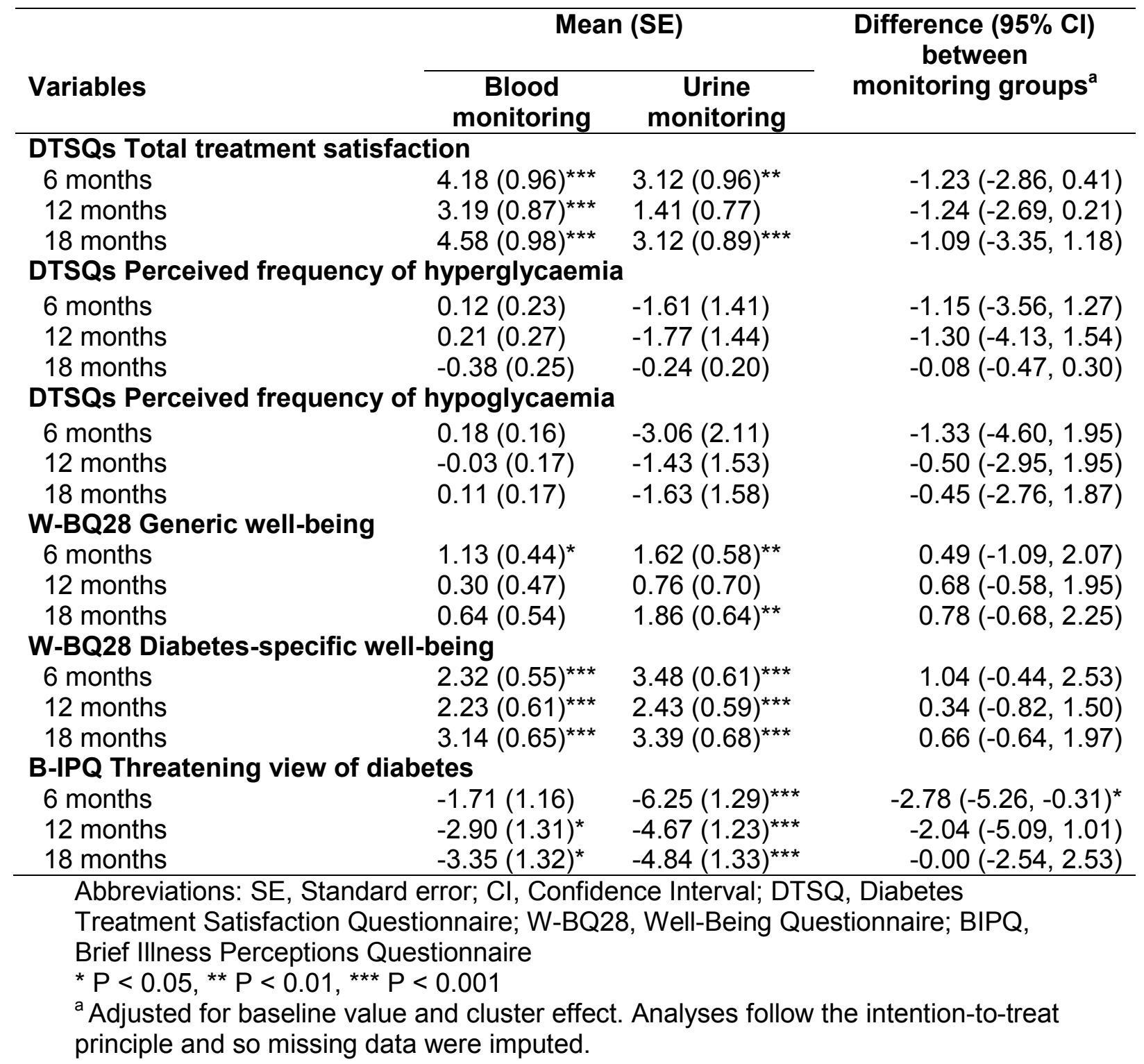


Figure 1. CONSORT diagram showing the flow of patients through the trial.

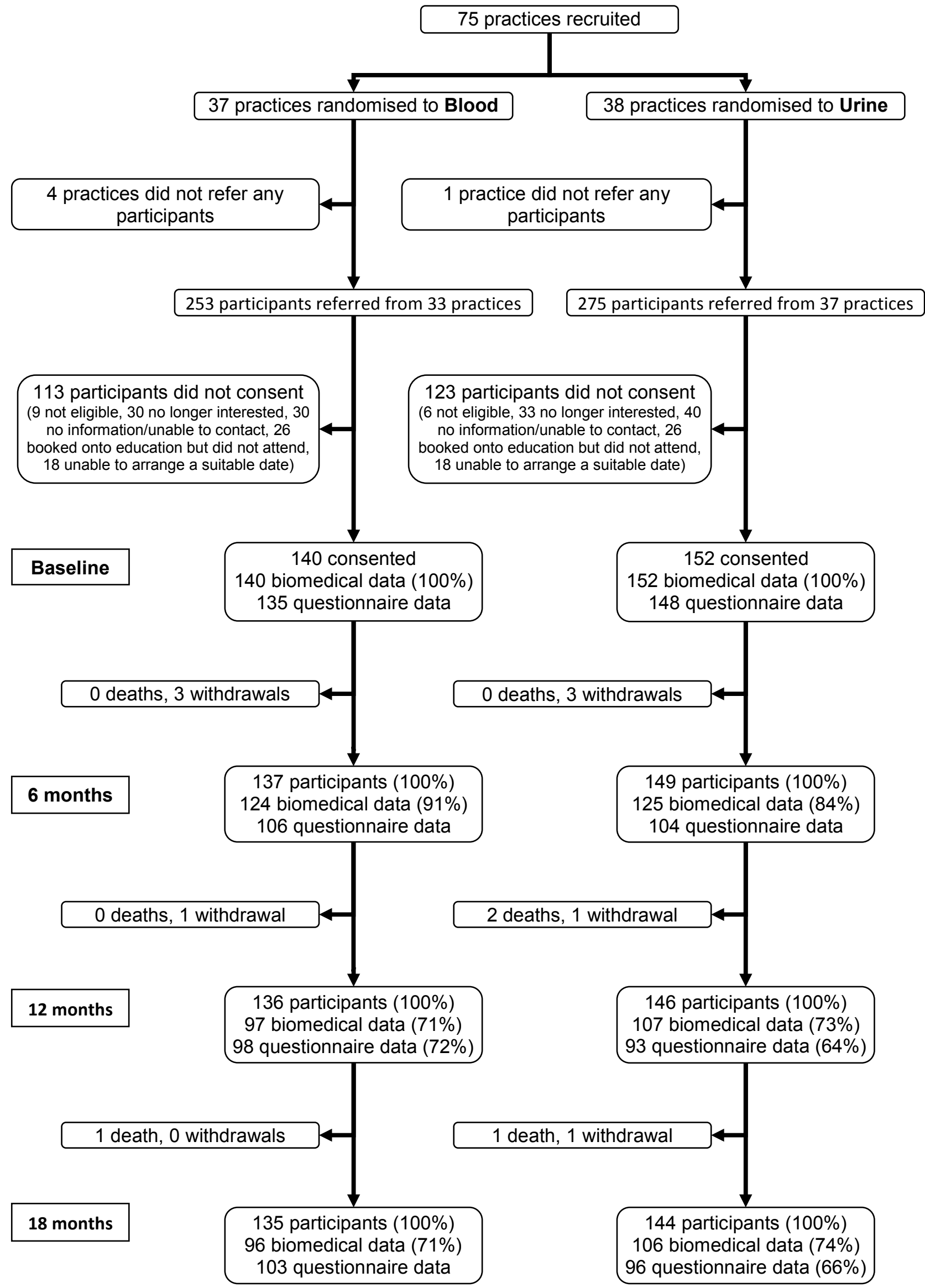


Figure 2. Self-reported monitoring method at each time point among participants who provided questionnaire data.

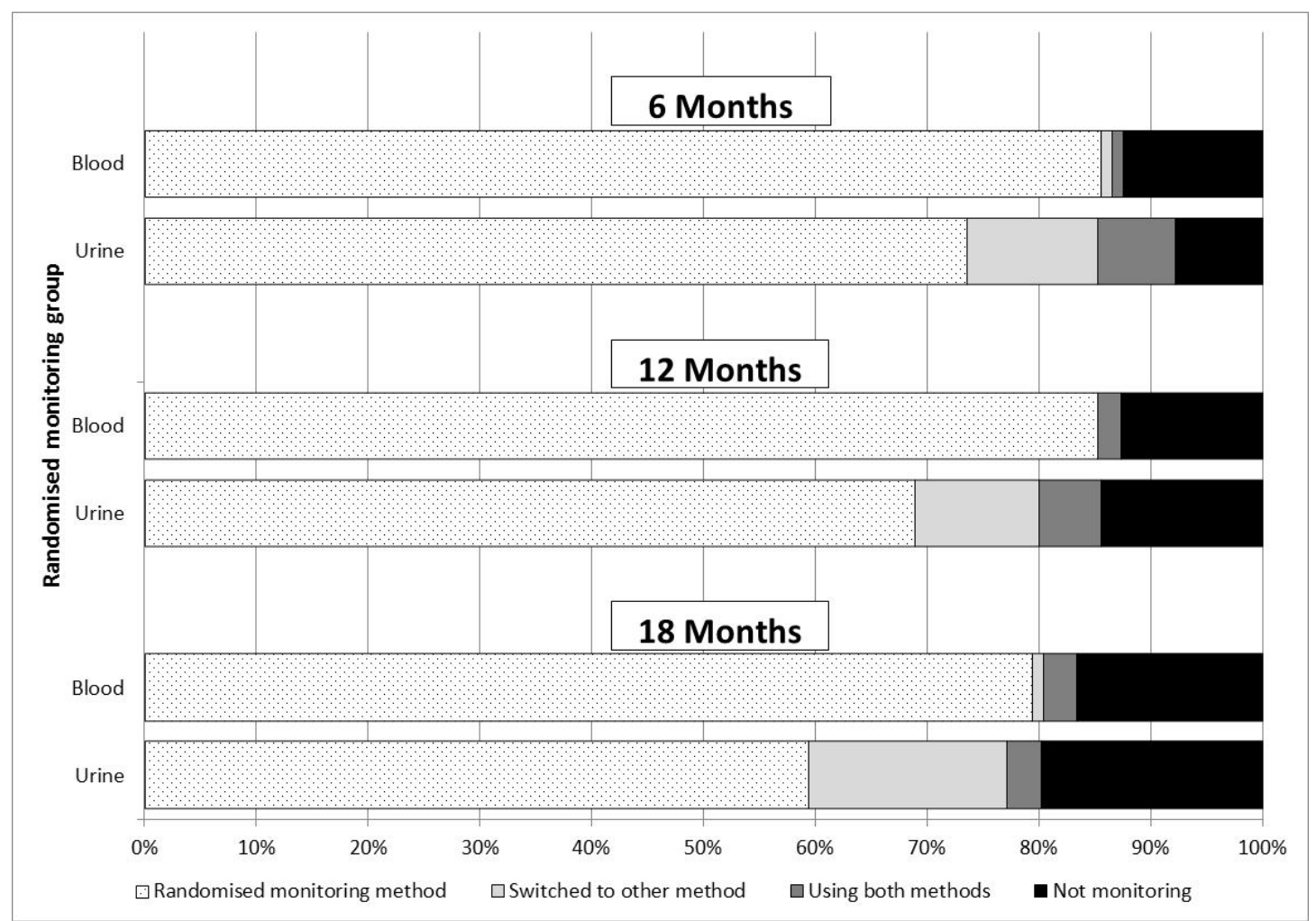


Supplementary Table 1. Sensitivity and per protocol analyses showing changes from baseline for biomedical outcomes.

\begin{tabular}{|c|c|c|c|c|}
\hline \multirow[b]{2}{*}{ Variables } & \multicolumn{2}{|c|}{ Complete data } & \multicolumn{2}{|c|}{ Per protocol } \\
\hline & $\mathbf{N}$ & $\begin{array}{l}\text { Difference } \\
(95 \% \mathrm{Cl}) \dagger\end{array}$ & $\mathbf{N}$ & $\begin{array}{l}\text { Difference } \\
(95 \% \mathrm{Cl}) \dagger\end{array}$ \\
\hline \multicolumn{5}{|c|}{ HbA1c, mmol/mol } \\
\hline 6 months & 235 & $-1(-3,1)$ & 164 & $0(-2,3)$ \\
\hline 12 months & 191 & $-1(-3,2)$ & 143 & $1(-4,2)$ \\
\hline 18 months & 188 & $-1(-4,1)$ & 138 & $0(-3,2)$ \\
\hline \multicolumn{5}{|l|}{ HbA1c, \% } \\
\hline 6 months & 235 & $-0.1(-0.3,0.1)$ & 164 & $0(-0.2,0.3)$ \\
\hline 12 months & 191 & $-0.1(-0.3,0.2)$ & 143 & $-0.1(-0.4,0.2)$ \\
\hline 18 months & 188 & $-0.1(-0.4,0.1)$ & 138 & $0(-0.3,0.3)$ \\
\hline \multicolumn{5}{|l|}{$B M I, k g / m^{2}$} \\
\hline 6 months & 195 & $0.2(-0.6,1.0)$ & 164 & $-0.4(-1.2,0.5)$ \\
\hline 12 months & 156 & $0.2(-0.7,1.0)$ & 143 & $0.1(-0.9,1.1)$ \\
\hline 18 months & 141 & $-0.2(-1.2,0.8)$ & 138 & $-0.4(-1.6,0.9)$ \\
\hline \multicolumn{5}{|c|}{ Waist circumference, cm } \\
\hline 6 months & 74 & $-0.5(-2.9,1.9)$ & 164 & $-1.44(-5.2,2.3)$ \\
\hline 12 months & 57 & $2.3(-3.0,7.6)$ & 143 & $-2.9(-6.8,0.9)$ \\
\hline 18 months & 35 & $-0.6(-9.5,8.3)$ & 138 & $-0.9(-4.2,2.4)$ \\
\hline \multicolumn{5}{|l|}{ Weight, kg } \\
\hline 6 months & 211 & $0.1(-1.8,2.1)$ & 164 & $-0.9(-3.2,1.4)$ \\
\hline 12 months & 173 & $0.1(-2.2,2.3)$ & 143 & $0.4(-1.8,2.7)$ \\
\hline 18 months & 154 & $-2.1(-5.3,1.1)$ & 138 & $-0.3(-3.9,3.4)$ \\
\hline \multicolumn{5}{|c|}{ Systolic BP, mmHg } \\
\hline 6 months & 195 & $-1.7(-5.5,2.0)$ & 164 & $-0.8(-5.7,4.1)$ \\
\hline 12 months & 125 & $0.6(-3.0,4.2)$ & 143 & $1.7(-3.1,6.5)$ \\
\hline 18 months & 96 & $-1.8(-6.0,2.5)$ & 138 & $-0.4(-4.6,3.8)$ \\
\hline \multicolumn{5}{|c|}{ Diastolic BP, mmHg } \\
\hline 6 months & 191 & $-1.2(-3.3,0.9)$ & 164 & $-0.8(-3.5,1.9)$ \\
\hline 12 months & 124 & $0.1(-2.5,2.7)$ & 143 & $0.2(-3.3,3.6)$ \\
\hline 18 months & 94 & $-1.8(-6.2,2.6)$ & 138 & $2.33(-1.2,5.9)$ \\
\hline \multicolumn{5}{|c|}{ Total cholesterol, mmol/l } \\
\hline 6 months & 202 & $-0.2(-0.5,0.1)$ & 164 & $-0.2(-0.5,0.1)$ \\
\hline 12 months & 161 & $0(-0.3,0.3)$ & 143 & $-0.1(-0.4,0.2)$ \\
\hline 18 months & 145 & $-0.1(-0.3,0.2)$ & 138 & $-0.1(-0.3,0.2)$ \\
\hline \multicolumn{5}{|c|}{ HDL cholesterol, mmol// } \\
\hline 6 months & 145 & $0(-0.1,0.1)$ & 164 & $0(-0.1,0.1)$ \\
\hline 12 months & 100 & $0.1(-0.1,0.3)$ & 143 & $0(-0.1,0.1)$ \\
\hline 18 months & 76 & $0(-0.1,0.1)$ & 138 & $-0.1(-0.2,0.1)$ \\
\hline \multicolumn{5}{|c|}{ LDL cholesterol, mmol// } \\
\hline 6 months & 132 & $-0.1(-0.4,0.2)$ & 164 & $-0.1(-0.4,0.2)$ \\
\hline 12 months & 93 & $-0.1(-0.4,0.2)$ & 143 & $-0.1(-0.4,0.3)$ \\
\hline 18 months & 68 & $0(-0.4,0.5)$ & 138 & $-0.1(-0.4,0.2)$ \\
\hline
\end{tabular}

Abbreviations: $\mathrm{Cl}$, Confidence Interval; BP, Blood Pressure; BMl, Body mass index

${ }^{*} \mathrm{P}<0.05,{ }^{* *} \mathrm{P}<0.01,{ }^{* * *} \mathrm{P}<0.001$

$\dagger$ Adjusted for baseline value and cluster effect. 
Supplementary Table 2. Sensitivity and per protocol analyses showing changes from baseline for psychosocial outcomes.

\begin{tabular}{|c|c|c|c|c|}
\hline \multirow[b]{2}{*}{ Variables } & \multicolumn{2}{|c|}{ Complete data } & \multicolumn{2}{|c|}{ Per protocol } \\
\hline & $\mathbf{N}$ & $\begin{array}{l}\text { Difference } \\
(95 \% \mathrm{Cl}) \dagger\end{array}$ & $\mathbf{N}$ & $\begin{array}{l}\text { Difference } \\
(95 \% \mathrm{Cl}) \dagger\end{array}$ \\
\hline \multicolumn{5}{|c|}{ DTSQs Total treatment satisfaction } \\
\hline 6 months & 136 & $-1.4(-3.3,0.5)$ & 164 & $-0.6(-2.6,1.4)$ \\
\hline 12 months & 133 & $-2.0(-3.6,-0.4)^{*}$ & 143 & $-1.8(-3.6,0.9)$ \\
\hline 18 months & 137 & $-2.0(-3.9,0)^{*}$ & 138 & $-1.3(-3.8,1.1)$ \\
\hline \multicolumn{5}{|c|}{ DTSQs Perceived frequency of hyperglycaemia } \\
\hline 6 months & 148 & $-1.9(-4.5,0.8)$ & 164 & $-0.7(-1.1,-0.3)^{* *}$ \\
\hline 12 months & 140 & $-2.2(-4.8,0.5)$ & 143 & $-0.7(-2.8,1.5)$ \\
\hline 18 months & 141 & $-0.2(-0.6,0.3)$ & 138 & $-0.4(-1.0,0.2)$ \\
\hline \multicolumn{5}{|c|}{ DTSQs Perceived frequency of hypoglycaemia } \\
\hline 6 months & 143 & $-3.3(-7.2,0.7)$ & 164 & $-1.4(-5.9,3.1)$ \\
\hline 12 months & 135 & $-1.4(-4.4,1.0)$ & 143 & $-0.3(-2.8,2.1)$ \\
\hline 18 months & 136 & $-1.7(-4.8,1.3)$ & 138 & $-2.1(-6.0,1.8)$ \\
\hline \multicolumn{5}{|c|}{ W-BQ28 12-item General well-being } \\
\hline 6 months & 170 & $0.6(-0.5,1.8)$ & 164 & $0.7(-0.7,2.0)$ \\
\hline 12 months & 158 & $1.1(-0.8,2.9)$ & 143 & $1.3(-0.6,3.3)$ \\
\hline 18 months & 165 & $1.5(0.2,2.8)^{*}$ & 138 & $1.6(-0.5,3.8)$ \\
\hline \multicolumn{5}{|c|}{ W-BQ28 Diabetes-specific well-being } \\
\hline 6 months & 176 & $2.0(0.3,3.7)^{*}$ & 164 & $1.5(-0.3,3.2)$ \\
\hline 12 months & 154 & $0.9(0,2.6)$ & 143 & $0.5(-1.4,2.5)$ \\
\hline 18 months & 167 & $0.9(-0.7,2.5)$ & 138 & $1.7(-0.0,3.5)$ \\
\hline \multicolumn{5}{|c|}{ B-IPQ Threatening view of diabetes } \\
\hline 6 months & 150 & $-5.1(-8.1-2.12)^{\star *}$ & 164 & $-4.8(-8.3,-1.4)^{* *}$ \\
\hline 12 months & 135 & $-2.7(-6.1,0.6)$ & 143 & $-3.5(-7.6,0.6)$ \\
\hline 18 months & 148 & $-1.6(-4.6,1.4)$ & 138 & $-2.3(-6.2,1.7)$ \\
\hline \multicolumn{5}{|c|}{$\begin{array}{l}\text { Abbreviations: } \mathrm{Cl} \text {, Confidence Interval; DTSQ, Diabetes Treatment Satisfaction } \\
\text { Questionnaire; W-BQ28, Well-Being Questionnaire; BIPQ, Brief Illness Perceptions } \\
\text { Questionnaire } \\
{ }^{*} \mathrm{P}<0.05,{ }^{* *} \mathrm{P}<0.01,{ }^{* * *} \mathrm{P}<0.001\end{array}$} \\
\hline
\end{tabular}

